\title{
Analysis of Writing Inks by Time-of-Flight Secondary Ion Mass Spectrometry - A Forensic Case Study
}

\author{
A. Schnieders, ${ }^{*}$ A. H. Lyter, III, ${ }^{* *}$ N. Havercroft, ${ }^{* * *}$ \\ * Tascon USA, Inc, 100 Red Schoolhouse Road, Bldg. A-8, Chestnut Ridge, NY 10977 \\ ** Federal Forensic Associates, Inc., P.O. Box 31567, Raleigh, NC 27622 \\ *** ION-TOF USA, Inc, 100 Red Schoolhouse Road, Bldg. A-8, Chestnut Ridge, NY 10977
}

The analysis of writing inks has garnered extensive interest by the forensic community in regards to differentiation, dating and the solving of problems such as line crossings and obliterations. Various assorted analytical techniques have been employed such as near infrared imaging, thin layer chromatography, chemical spot tests, high performance liquid chromatography, gas chromatography/mass spectrometry, Raman spectroscopy and capillary electrophoresis.

This presentation will evaluate the capabilities of surface mass spectrometry and especially Time-ofFlight Secondary Ion Mass Spectrometry (ToF-SIMS) in the analysis of writing inks. ToF-SIMS has besides its superb surface sensitivity several advantages that will be elucidated, including superior mass resolution needed for chemical identification of inks, minimal sample destruction, and imaging capabilities. Because of the limited destruction ToF-SIMS is a desirable examination technique in those instances where the evidence is either extremely limited, as in the case of trace evidence, or extremely valuable, as in the case of antiquities or historical documents. The imaging capabilities of ToF-SIMS also allow this examination technique to be used in a court setting where examination results can be easily demonstrated to the trier of fact. One such instance where this circumstance arises is that of a "line crossing". The term "line crossing" refers to two intersecting lines, prepared by either writing or printing. Historically, microscopic examination has been the technique of choice for this problem, but high instances of inconclusive results and lack of ability to conclusively illustrate results has caused continuous research for a better solution. This work will illustrate a series of differences detected by ToF-SIMS among a group of ink formulations. These differences in mass spectra, combined with the surface sensitivity, allow for points of comparison that can be used to determine the sequence of application in a "line crossing" problem. FIG. 1. shows a three color overlay of maps of characteristic secondary ions for the paper and the two inks used for the signatures. It can be clearly seen that signature 1 was set later than signature 2 . 


\section{Signature 1}

Signature 2
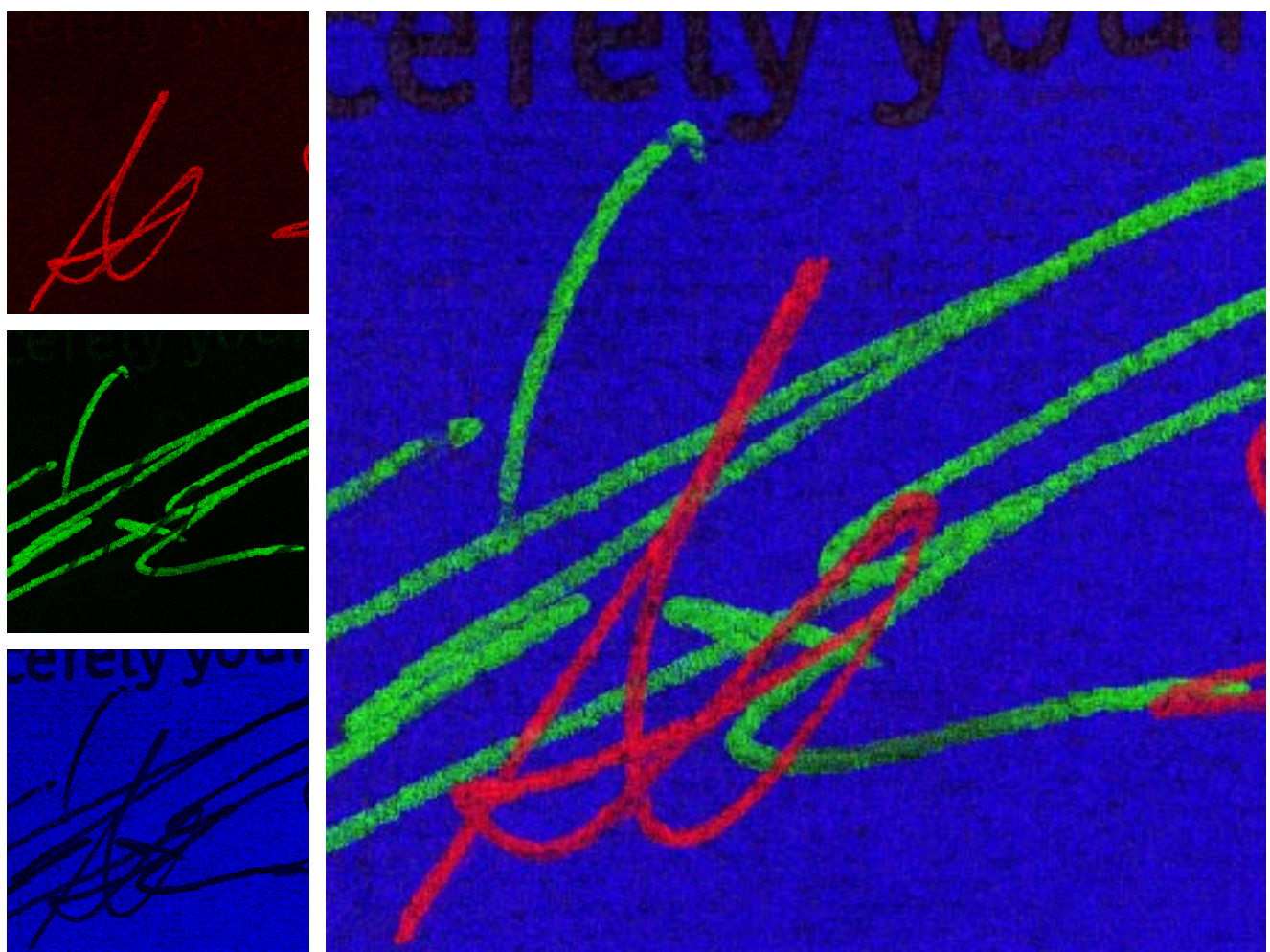

FIG. 1. Chemical maps of characteristic secondary ions for the paper and the two inks used for the signatures and 3-color overlay of the maps. Field of View $15 \times 15 \mathrm{~mm}^{2}$. 